\title{
Análise Macroscópica Infra-Vermelha da Difusão do Óxido Nitroso Via Inalatória para a Cavidade Abdominal, em Ratos Submetidos a Pneumoperitônio *
}

\section{Macroscopic Infrared Analysis of Inhaled Nitrous Oxide Diffusion to Abdominal Cavity in Rats Submitted to Pneumoperitoneum}

\author{
Daniel Colman ${ }^{1}$; Marcos Leal Brioschi ${ }^{2}$; Mário Cimbalista Júnior ${ }^{3}$; Elizabeth Mila Tambara, TSA ${ }^{4}$;
} Maria Célia Barbosa Fabrício de Melo, TSA ${ }^{5}$; Leonardo Pimpão Blume ${ }^{6}$

\section{RESUMO}

Colman D, Brioschi ML, Cimbalista Jr M, Tambara EM, Melo MCBF, Blume LP - Análise Macroscópica Infra-Vermelha da Difusão do Óxido Nitroso Via Inalatória para a Cavidade Abdominal, em Ratos Submetidos a Pneumoperitônio

\begin{abstract}
JUSTIFICATIVA E OBJETIVOS: O óxido nitroso $\left(\mathrm{N}_{2} \mathrm{O}\right)$, por ser uma estrutura tri-atômica assimétrica, assume características de alta emissão e absorção de energia no espectro infra-vermelho, com um pico característico de absorção em 4,5 um, o que o torna visível ao infra-vermelho curto, quando contrastado com uma fonte emissora de calor (anteparo quente). Diversos autores têm descrito a difusão do $\mathrm{N}_{2} \mathrm{O}$ para cavidades fechadas por métodos como cromatografia gasosa e analisador de gases, que não permitem um estudo macroscópico detalhado do gás. O presente estudo teve como objetivo a filmagem macroscópica no espectro infra-vermelho da difusão de $\mathrm{N}_{2} \mathrm{O}$, utilizado em anestesia inalatória, para a cavidade peritoneal de ratos submetidos a pneumoperitônio de $20 \mathrm{mmHg}$ com ar ambiente.
\end{abstract}

MÉTODO: Dividiu-se os animais em três grupos, de acordo com o anestésico utilizado: I - controle venoso: tiopental intra-peritoneal; II - controle inalatório: isoflurano a 1,2\% em $\mathrm{O}_{2}$ 100\%; III - óxido nitroso: $\mathrm{N}_{2} \mathrm{O} 66 \%$ em oxigênio e isoflurano a $0,6 \%$. Os termogramas provenientes da descompressão abdominal foram obtidos, por meio de um radiômetro AGEMA 550 filmados a 7 quadros por segundo.

\footnotetext{
* Recebido do (Received from) Grupo de Pesquisas em Imagem Infravermelha, CNPq/PUC/PR. Realizado no Laboratório de Lesões Medulares e Trauma Experimental da Pontifícia Universidade Católica do Paraná (PUC/PR). Artigo vencedor do prêmio Renato Ângelo Saraiva de 2002

1. Médico Anestesiologista do Hospital de Clínicas da UFPR. Mestrando em Clínica Cirúrgica pela UFPR

2. Mestre em Princípios da Cirurgia - Faculdade Evangélica de Medicina do Paraná. Doutorando em Clínica Cirúrgica pela UFPR. Professor da disciplina de Anatomia Médica da PUC/PR e UFPR. Presidente da Sociedade Internacional de Termografia (UKTA/ITA)

3. Engenheiro Eletricista, Diretor Técnico Thermotronics ST Ltda. Membro do IEEE - Engineering in Medicine na Biology Society

4. Doutora em Clínica Cirúrgica pela UFPR. Responsável pelo CET/SBA do HC da UFPR. Professora Titular da disciplina de Anestesiologia da PUC/PR. Professora Adjunta de Anestesiologia da UFPR

5. Doutora em Clínica Cirúrgica pela UFPR. Responsável pelo CET/SBA da Santa Casa de Misericórdia de Curitiba. Professora Adjunta da Disciplina de Anestesiologia da PUC/PR

6. Graduando em Medicina pela PUC/PR.
}

Apresentado (Submitted) em 30 de novembro de 2002 Aceito (Accepted) para publicação em 25 de julho de 2003

Endereço para correspondência (Correspondence to) Dr. Daniel Colman

Rua Desembargador Westphalen 824/704-B Rebouças 80220-030 Curitiba, PR

(C) Sociedade Brasileira de Anestesiologia, 2004
RESULTADOS: $\mathrm{O} \mathrm{N}_{2} \mathrm{O}$ demonstrou-se visível ao infra-vermelho. No momento da descompressão abdominal, não houve nos grupos I e II termogramas com rastros de gases visíveis ao infra-vermelho. Houve, porém, rastros de gases visíveis ao infra-vermelho no grupo III.

CONCLUSÕES: Conclui-se que o óxido nitroso inalatório a $66 \%$ difundiu-se para a cavidade peritoneal de ratos submetidos a pneumoperitônio de $20 \mathrm{mmHg}$ com ar ambiente, sem aumento de pressão intra-abdominal.

Unitermos: ANESTÉSICOS, Gasoso: óxido nitroso; ANIMAL: rato; TÉCNICAS DE MEDIÇÃO: imagem infra-vermelha

\section{SUMMARY}

Colman D, Brioschi ML, Cimbalista Jr M, Tambara EM, Melo MCBF, Blume LP - Macroscopic Infrared Analysis of Inhaled Nitrous Oxide Diffusion to Abdominal Cavity in Rats Submitted to Pneumoperitoneum

BACKGROUND AND OBJECTIVES: Nitrous oxide $\left(\mathrm{N}_{2} \mathrm{O}\right)$, for its tri-atomic asymmetric structure, has high energy emission and absorption characteristics within the infrared spectrum, with maximum absorption at $4.5 \mu \mathrm{m}$, what makes it visible at short infrared, when contrasted with a heat emission source (hot support). Many authors have described $\mathrm{N}_{2} \mathrm{O}$ diffusion to closed cavities by chromatography methods and gas analyzers, which do not allow a detailed macroscopic study of the gas. This study aimed at macroscopically filming in the infrared spectrum inhaled $\mathrm{N}_{2} \mathrm{O}$ diffusion to the peritoneal cavity of rats submitted to $20 \mathrm{mmHg}$ room air pneumoperitoneum.

METHODS: Animals where divided in three groups according to the anesthetic drug: I - Intravenous control: intraperitoneal thiopental; II - inhaled control: $1.2 \%$ isoflurane in $100 \% \mathrm{O}_{2}$; III nitrous oxide: $66 \% \mathrm{~N}_{2} \mathrm{O}$ in oxygen and $0.6 \%$ isoflurane. Thermal images of abdominal decompression where captured by an A GEMA 550 radiometer filmed at 7 frames per second.

RESULTS: $\mathrm{N}_{2} \mathrm{O}$ was visible to infrared. At abdominal decompression, groups I and II have not shown visible gas traces at infrared thermographs, while group III had visible infrared traces.

CONCLUSIONS: Our conclusion was that $66 \%$ inhaled nitrous oxide has diffused to peritoneal cavity of rats submitted to 20 $\mathrm{mmHg}$ room air pneumoperitoneum, with no intra-abdominal pressure increase.

Key Words: ANESTHETICS, Gaseous: nitrous oxide ANIMAL: rat; MEASUREMENT TECHNIQUES: infrared image

\section{INTRODUÇÃO}

$\mathrm{O}$ xido nitroso $\left(\mathrm{N}_{2} \mathrm{O}\right)$, o mais antigo dos agentes inalatórios, é amplamente utilizado no mundo todo ${ }^{1}$. Suas propriedades, em especial a baixa solubilidade ${ }^{2}$, conferem características farmacocinéticas especiais e desejáveis a 
um agente inalatório. Os processos de captação, distribuição e eliminação são muito rápidos. Apresenta ações colaterais pouco pronunciadas, merecendo destaque as mínimas repercussões cardiovasculares e respiratórias ${ }^{3,4}$. Além disso, é pouco metabolizado.

Do ponto de vista estrutural, o $\mathrm{N}_{2} \mathrm{O}$ é uma molécula tri-atômica assimétrica, possuindo características de grande absorção e emissão de irradiação infra-vermelha ${ }^{5}$, com um pico característico em $4,5 \mu \mathrm{m}$ no espectro. Pelo fato de ser empregado em altas concentrações na anestesia inalatória e largamente utilizado em pediatria ${ }^{6}$, foi escolhido como modelo dos estudos macroscópicos de gases envolvidos na exposição ocupacional do anestesiologista. Desse modo, adiciona uma nova dimensão no estudo da dispersão de gases anestésicos no centro cirúrgico e na avaliação de sistemas de drenagem do ar da sala de operações ${ }^{7}$, bem como na adaptação da máscara facial de maneira correta a evitar vazamentos, na produção de material didático- educativo, servindo ainda como um indicador de gases e vapores perdidos no ambiente, tanto quantitativamente, quanto qualitativamente ${ }^{8}$.

Algumas controvérsias permanecem sobre o uso de $\mathrm{N}_{2} \mathrm{O}$ em Anestesiologia: graças à sua solubilidade, maior que a do nitrogênio ${ }^{9}$, passa a ocupar espaços antes preenchidos por este em cavidades do organismo preenchidas por gases (como o ouvido médio) ${ }^{10-12}$, aumentando tanto em volume quanto em pressão em seu interior. Apesar de seu potencial para uso em laparoscopia como agente inalatório ou no próprio pneumoperitônio ${ }^{13}$, o $\mathrm{N}_{2} \mathrm{O}$ pode contribuir para distensão de alças intestinais ${ }^{14}$, apesar de que este efeito tem sido contestado em estudos mais recentes. Spivak e col., em cirurgias laparoscópicas, não evidenciaram diferença significativa entre o uso de $\mathrm{N}_{2} \mathrm{O}$ ou de ar atmosférico em relação às condições do campo cirúrgico ${ }^{15}$

O presente estudo tem como objetivo analisar macroscopicamente a difusão do óxido nitroso, utilizado em anestesia inalatória, para a cavidade peritoneal, através da filmagem no espectro infra-vermelho em ratos submetidos a pneumoperitônio com pressão de $20 \mathrm{mmHg}$ de ar ambiente.

\section{MÉTODO}

O protocolo experimental utilizado neste estudo foi aprovado pelo Centro de Ciências Biológicas e da Saúde da Pontifícia Universidade Católica do Paraná (CCBS-PUC/PR) e conduzido obedecendo aos princípios éticos do Colégio Brasileiro de Experimentação Animal (COBEA) e registrado no Grupo de Pesquisas em Imagem Infra-vermelha, do Conselho Nacional de Pesquisas - PUC/PR.

Foram utilizados 15 ratos machos da linhagem Wistar (Rattus norvegicus albinus, Rodentia mammalia), com idades entre 120 e 153 dias (média de 135,9 dias). No pré-operatório forneceu-se ração padronizada e água ad libitum, até 12 horas antes da anestesia.

O experimento foi realizado no Laboratório de Lesão Medular e Trauma Experimental da PUC/PR. Admitiu-se mínima variação térmica, com temperatura ambiental mantida em $20^{\circ} \mathrm{C}$ e umidade relativa do ar em $75 \%$. As mesmas foram verificadas com termo-higrômetro de bulbo seco e úmido (Incotherm, Br). As perdas de calor por convecção foram minimizadas mantendo-se portas e janelas fechadas e mínima movimentação ao redor dos animais. O fluxo de ar foi controlado com um anemômetro digital de pás rotativas, modelo HHF 300 A (Omega Engineering, Inc), a uma distância de 10 $\mathrm{cm}$ do animal, mantendo-se a velocidade do fluxo de ar menor que $0,2 \mathrm{~m} \cdot \mathrm{s}^{-1}$. Este é o valor de transição entre a perda de calor por convecção livre e forçada ${ }^{16}$.

A anestesia foi induzida e mantida com os agentes estudados: tiopental sódico, isoflurano e isoflurano associado ao $\mathrm{N}_{2} \mathrm{O}$ a $66 \%$ ) sendo os agentes anestésicos inalatórios administrados através de um cone facial com oxigênio e uso de vaporizador universal com fluxo de admissão de gases de 3 L. $\min ^{-1}$.

Os animais foram mantidos em plano anestésico nível III de Guedel. A monitorização foi realizada observando-se a presença de reflexos, a freqüência respiratória, a coloração das mucosas ${ }^{17}$ e as alterações da pressão intra-abdominal. Os animais foram divididos em 3 grupos:

- Grupo I - controle venoso: anestesiados com tiopental por via peritoneal e uso de cone facial com oxigênio a 3 L. $\min ^{-1}$;

- Grupo II - controle inalatório: anestesiados com isoflurano a $1,2 \%$ em cone facial com oxigênio a 3 L. $\mathrm{min}^{-1}$;

- Grupo III - óxido nitroso: anestesiados com isoflurano a $0.6 \%$ em cone facial com oxigênio a $1 \mathrm{~L} \cdot \mathrm{min}^{-1}$ associado a $\mathrm{N}_{2} \mathrm{O}$ a 2 L. $\min ^{-1}(66,6 \%)$.

Na saída do vaporizador universal de gases foi acoplado um tubo plástico em forma de "T" que desviou o fluxo em duas direções, uma para o cone facial em contato com o animal para indução e manutenção da anestesia, e outra para controle dos gases do sistema anestésico e filmagem.

Para a obtenção do pneumoperitônio ${ }^{18} \mathrm{com}$ pressão intra-abdominal de $20 \mathrm{mmHg}$, puncionou-se a cavidade peritoneal com cateter de teflon $22 \mathrm{G}$ conectado a um sistema de anerômetro (Welch Allyn, Tycos ${ }^{\circledR}$, Arden, USA) previamente calibrado. O sistema permaneceu acoplado à cavidade abdominal, permitindo monitorização contínua da pressão intra-abdominal. Uma vez obtida a pressão desejada, interrompeu-se a insuflação. Após 45 minutos, procedeu-se à descompressão do pneumoperitônio por meio de nova punção abdominal com cateter de teflon $16 \mathrm{G}$ e filmagem da saída do gás contido na cavidade abdominal no espectro infra-vermelho compreendido entre 3,5 a $5 \mu \mathrm{m}$. Esta nova punção foi realizada com a finalidade de se obter um esvaziamento mais rápido. Durante a descompressão abdominal, suspendeu-se a administração de gases anestésicos ao animal, para evitar contaminação com $\mathrm{N}_{2} \mathrm{O}$ durante a filmagem.

Um radiômetro Thermovision AGEMA 550 (FLIR Systems ${ }^{\mathrm{TM}}$, Suécia) foi utilizado para captar a faixa espectral das ondas eletromagnéticas emitidas entre 3,5 e $5 \mu \mathrm{m}$, isto é, ondas no espectro infra-vermelho curto. A resolução espacial máxima obtida é de 0,1 a 0,2 $\mathrm{mm}$. 
A irradiação infra-vermelha que é emitida naturalmente por objetos no ambiente é captada e convertida, por meio de um detector de PtSi resfriado com nitrogênio líquido (steerling cicle), em sinal elétrico. Este sinal é processado em uma planilha numérica de 76.000 pontos de temperatura absoluta, calibrados por quadro, representada instantaneamente em imagem térmica com resolução de $320 \times 240$ pixels e sensibilidade térmica maior que $0,1^{\circ} \mathrm{C}$. Os gases tri-moleculares, inclusive o $\mathrm{N}_{2} \mathrm{O}$, têm a propriedade física de absorção e emissão de energia na faixa infra-vermelha ${ }^{4,19}$, o que o torna visível ao infra-vermelho, quando colocado entre um anteparo que emite calor e um radiômetro.

Neste estudo, utilizou-se uma caixa de acrílico contendo água a $40^{\circ} \mathrm{C}$ como fonte emissora de calor para visualização do óxido nitroso ao infra-vermelho.

O radiômetro foi montado em um suporte vertical a $1 \mathrm{~m}$ de distância da caixa de acrílico, focada diretamente na superfície ventral do animal de modo a formar um ângulo de $60^{\circ} \mathrm{com} o$ cateter de teflon, durante a filmagem da descompressão abdominal. Filmou-se também, a título de controle, os gases que estavam sendo administrados ao animal via cone facial, pela saída em "T" proveniente do sistema de anestesia, conforme a figura 1.

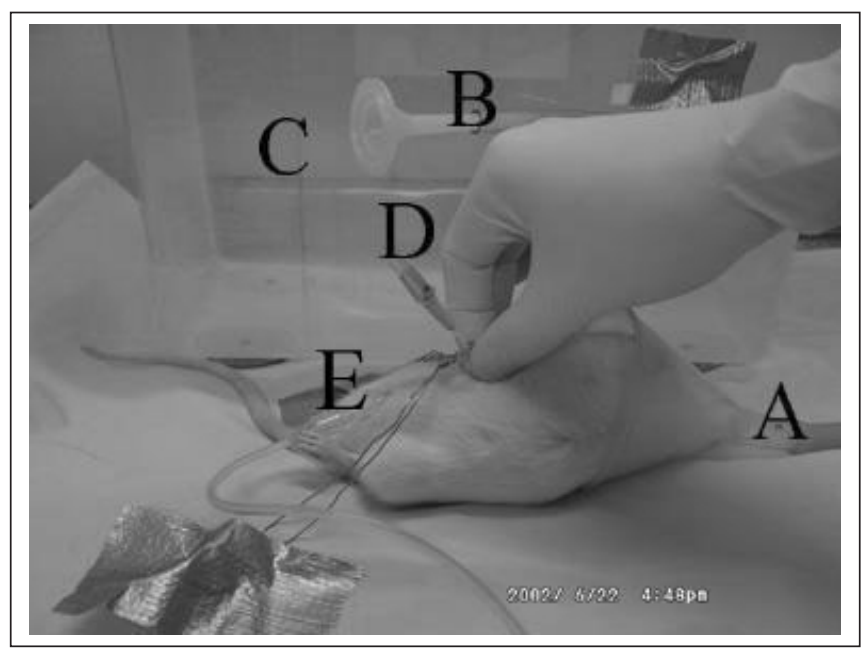

Figura 1 - Montagem do Experimento

A) Cone facial onde o anestésico foi administrado ao animal; B) Controle dos gases anestésicos que eram administrados ao animal; C) Caixa de acrílico contendo água aquecida em seu interior, servindo de anteparo para filmagem no infra-vermeIho; D) Cateter de teflon para descompressão abdominal; E) sistema de anerômetro conectado à cavidade abdominal para obtenção de pneumoperitônio e monitorização de pressão intra-abdominal

As imagens foram processadas em computador Pentium III $750 \mathrm{MHz}$ acoplado a uma placa PCMCIA. Por meio de um programa específico, ThermaCAM ${ }^{\mathrm{TM}}$ Researcher 2001, FLIR Systems (Suécia), as imagens capturadas foram gravadas a 7 quadros por segundo durante todo o momento da descompressão abdominal, nas mesmas condições ambientais já descritas. Todas as imagens foram representadas por termogramas infra-vermelhos em um monitor de vídeo e gravadas em CD-Rom para posterior análise pelo programa.
As imagens foram plotadas utilizando-se amplitude térmica (range)entre $50^{\circ} \mathrm{Ce} 20^{\circ} \mathrm{C}$, temperatura média (level) de $42^{\circ} \mathrm{C}$ e escala colorimétrica (pallete) contínua "RAINBOW900", na qual as cores branco, vermelho, amarelo, verde, azul e preto representaram um gradiente decrescente das áreas de temperatura, igualmente distribuídas na escala, da mais quente para mais fria e mantida até o final do experimento. Para efeitos de impressão em preto e branco, as imagens foram trabaIhadas com o software Adobe Photoshop 5.0, através do qual as cores foram filtradas na palheta vermelha seguido de aplicação de tons de cinza para um melhor contraste entre os elementos da pesquisa.

Para análise semi-quantitativa de gases, visíveis ao infra-vermelho, calculou-se o diferencial de temperatura entre o controle do que o animal estava inalando (item B da figura 1) com o que saiu da cavidade abdominal (item D da figura 1).

\section{RESULTADOS}

O óxido nitroso a $66 \%$ pode ser visualizado ao infra-vermelho pelo método descrito e protocolado em termogramas, em que cores amareladas representam a emissão de calor pelo anteparo de acrílico com água aquecida em seu interior, e as cores esverdeadas (mais frias) representam a energia absorvida pelo $\mathrm{N}_{2} \mathrm{O}$, formando um contraste visível à filmagem infra-vermelha, conforme a figura 2.

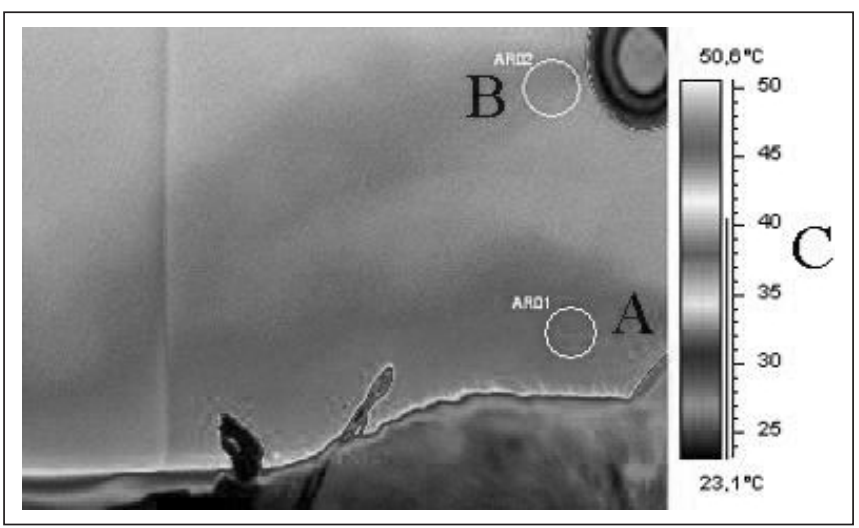

Figura 2 - A) Gases Provenientes do Cone Facial

B) Gases provenientes do controle de A; C) Escala colorimétrica. Em A e B os gases são visíveis ao infra-vermelho

No grupo I não houve formação de imagens de rastro de gases visíveis ao infra-vermelho nos termogramas obtidos durantea filmagem macroscópica dos gases provenientes do cone facial $\left(\mathrm{O}_{2}\right.$ a $\left.100 \%\right)$, conforme a figura $3 . \mathrm{O}_{2}$ demonstrou-se invisível ao infra-vermelho. Também não houve a formação de imagens de rastros de gases nos termogramas obtidos durante a filmagem macroscópica dos gases provenientes da cavidade intra-abdominal, durante a descompressão, conforme a figura 4 .

Revista Brasileira de Anestesiologia Vol. 54, № 2, Março - Abril, 2004 


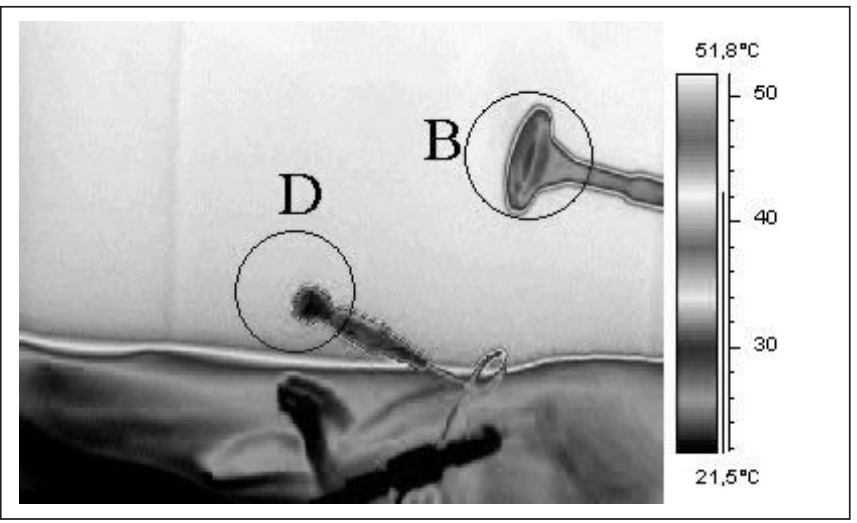

Figura 3 - Pré-Descompressão Abdominal

B) Gás não visível ao infra-vermelho

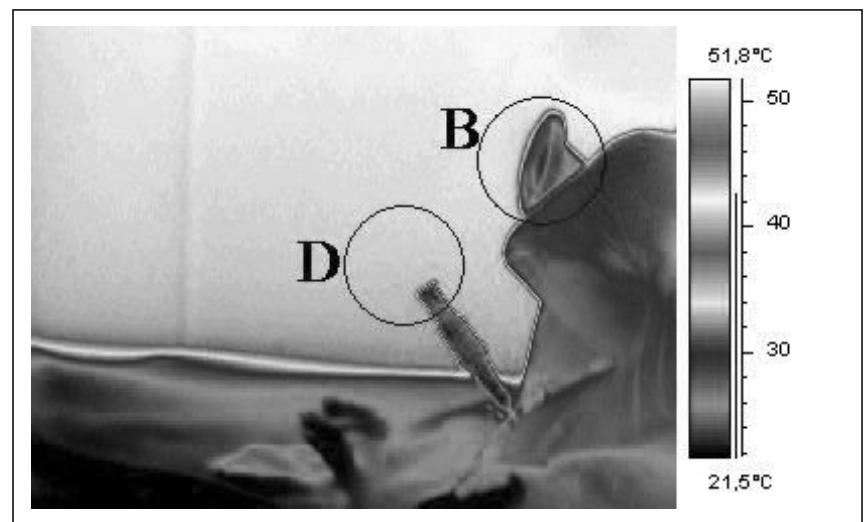

Figura 4 - Durante Descompressão Abdominal

B e D são invisíveis ao infra-vermelho

Em relação ao grupo II não houve formação de imagens de rastros de gases visíveis ao infra-vermelho em termogramas provenientes durante a filmagem macroscópica dos gases provenientes do cone facial $\left(\mathrm{O}_{2}\right.$ a $100 \%$ carreando isoflurano a $1,2 \%$ ). Neste estudo, com o método empregado e a faixa espectral utilizada, o isoflurano demonstrou-se invisível ao infra-vermelho. Também não houve formação de imagens de rastros de gases visíveis ao infra-vermelho em termogramas provenientes dos gases da cavidade intra-abdominal durante a descompressão. O padrão dos termogramas obtidos no grupo II seguem os padrões das figuras 3 e 4 .

No grupo III houve formação de imagens de rastros de gases visíveis ao infra-vermelho em termogramas provenientes durante a filmagem macroscópica dos gases provenientes do cone facial $\left(\mathrm{O}_{2}\right.$ a $33,4 \%$ associado a $\mathrm{N}_{2} \mathrm{O}$ a $66,6 \%$ com isoflurano a $0,6 \%$ ). Isso demonstra claramente que o $\mathrm{N}_{2} \mathrm{O}$ é visível em termogramas obtidos na faixa infra-vermelha, seguin- do-se o padrão do figura 2. Imagens com padrão de gases visíveis ao infra-vermelho foram obtidas também durante a filmagem macroscópica dos gases provenientes da cavidade intra-abdominal, durante a descompressão, conforme as figuras 5 e 6 . O diferencial de temperatura entre $B$ da figura $2 \mathrm{e}$ $D$ das figuras 5 e 6 foi próximo a zero, sugerindo que a concentração de $\mathrm{N}_{2} \mathrm{O}$ que saiu da cavidade era próxima da fração inspirada pelo animal, ou seja, $66 \%$.

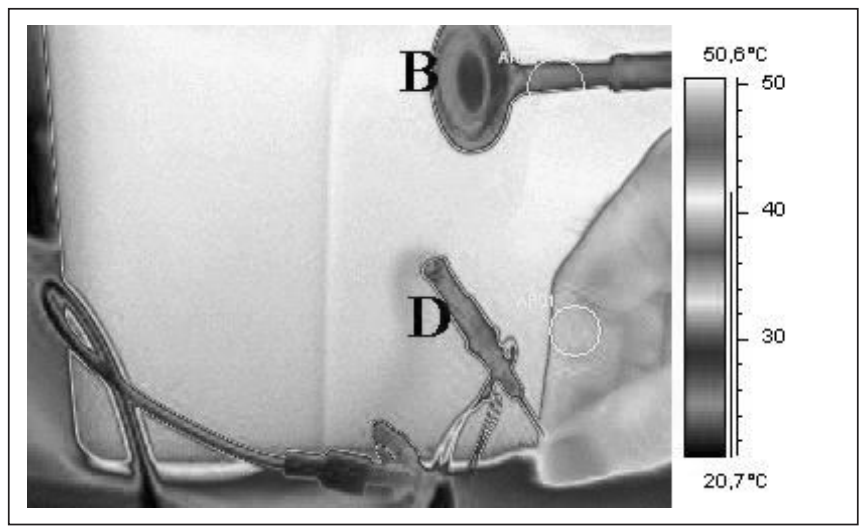

Figura 5 - Durante a Descompressão Abdominal no Grupo III B) Padrão de gás não visível ao infra-vermelho, pois foi suspensa a administração de $\mathrm{N}_{2} \mathrm{O}$ durante a descompressão abdominal. D) Padrão de gás visível ao infra-vermelho

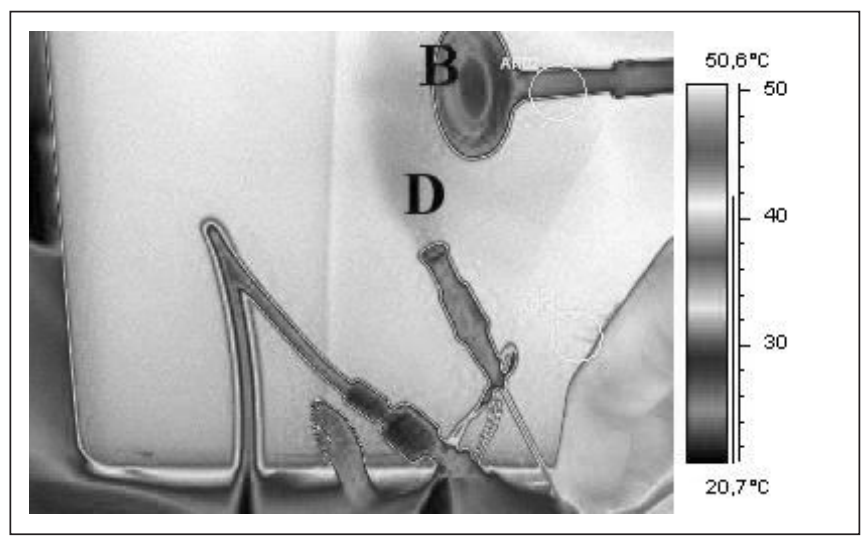

Figura 6 - Descompressão Abdominal com Padrão de Gás Visível ao Infra-Vermelho, Proveniente da Cavidade Peritoneal (D)

Tal fato demonstra a difusão do óxido nitroso utilizado na anestesia geral inalatória para cavidade peritoneal, em ratos submetidos a pneumoperitônio com $20 \mathrm{mmHg}$ de ar ambiente.

A pressão intra-abdominal manteve-se estável em $20 \mathrm{mmHg}$ durante o estudo, nos três grupos. 


\section{DISCUSSÃO}

A maioria dos gases e vapores com estrutura molecular bipolar absorve energia infra-vermelha. Se tal gás for interposto entre um objeto emitindo radiação infra-vermelha e um radiômetro, o gás absorverá a radiação infra-vermelha e esta energia absorvida se traduzirá como um abaixamento na temperatura detectada no termovisor, sendo visualizado na análise do termograma ${ }^{19}$. Por este método é possível visualizarem-se gases invisíveis ao olho humano.

Este métod o foi utilizado para o estudo dos níveis de poluição de agentes inalatórios mais comumente utilizados em Anestesiologia e permite o estudo das dispersões de gases poluentes e vapores durante o exercício profissional. Um estudo pesquisou níveis de poluição em salas de um serviço de anestesia pediátrica e demonstrou a importância de manuseio correto da máscara facial para se evitarem perdas de gases anestésicos poluentes para o meio ambiente. Concluiu que este método, além de permitir uma análise macroscópica da difusão do gás no ambiente, propicia ainda uma análise semi-quantitativa que teve correlação com os dados obtidos pelo analisador de gases ${ }^{19}$. O mesmo autor concluiu que os gases poluentes são insuficientemente evacuados da sala cirúrgica durante a ventilação. Neste estudo, além de se analisar macroscopicamente a difusão de $\mathrm{N}_{2} \mathrm{O}$ por via inalatória para o pneumoperitônio, pode-se inferir que sua concentração era próxima à fração inspirada, por meio da análise de subtração de imagens.

Aatmosfera possui certas propriedades de emissão e absorção de energia. As moléculas de gases e vapores vibram em determinadas freqüências ${ }^{5}$, e quando atingidas, absorvem um fóton de energia, o que faz com que um gás possa vir a ser contrastado quando passa entre a fonte emissora e o radiômetro.

Os gases monoatômicos, como o Neon, fazem somente transições eletrônicas, enquanto que gases diatômicos, como o $\mathrm{O}_{2}$ e o $\mathrm{N}_{2}$, não possuem momento elétrico dipolo, e portanto não absorvem nem emitem energia significativamente em condições ambientais, sendo considerados invisíveis ao infra-vermelho ${ }^{5}$. Tal fato foi observado em nosso estudo, pois não se evidenciou em nenhum momento o aparecimento de imagem de gás visível ao infra-vermelho quando $\circ \mathrm{O}_{2}$ foi usado no grupo I.

O pneumoperitônio foi realizado com ar atmosférico, sabidamente invisível ao infra-vermelho.

Moléculas assimétricas diatômicas, como o NO e o CO, emitem e absorvem energia infra-vermelha, mas de maneira muito fraca em condições ambientais. Já moléculas tri-atômicas como o $\mathrm{N}_{2} \mathrm{O}, \mathrm{SO}_{2}, \mathrm{H}_{2} \mathrm{O}$, e $\mathrm{CO}_{2}$ exibem fortes tendências para absorção ou emissão de energia. Estes gases podem vir a atrapalhar a interpretação dos componentes da imagem encontrada, pois o radiômetro utilizado neste estudo possui uma janela de observação na faixa 3,5 a $5 \mu \mathrm{m}$, enquanto tais moléculas possuem janelas com o pico de absorção nas seguintes faixas ${ }^{5}: \mathrm{CO}_{2}(2,0 ; 2,7,4,3 ; 15 \mu \mathrm{M}) ; \mathrm{H}_{2} \mathrm{O}(1,4 ; 1,9 ; 2,7$; $6,320 \mu \mathrm{M})$ e $\mathrm{N}_{2} \mathrm{O}(4,5 \mu \mathrm{M})$. Tal fenômeno pode ser controlado em nossos estudos utilizando-se ar ambiente no pneumoperitônio, que é sabidamente invisível ao infra-vermelho. No grupo II, sabendo que o $\mathrm{O}_{2}$ é invisível ao infra-vermelho, as imagens obtidas com isoflurano (como controle do que era vaporizado), demonstraram que este agente mostrou-se também invisível nas condições deste estudo. Uma vez que não houve aparecimento de rastro de gás visível ao infra-vermelho ao se descomprimir o abdômen nos animais do grupo II, torna-se improvável que o vapor d'água oriundo da serosa peritoneal contido na cavidade abdominal venha a exercer influência nos resultados encontrados nas condições deste estudo.

Em relação ao grupo III, no qual se utilizou $\mathrm{N}_{2} \mathrm{O}$ por via inalatória com isoflurano e $\mathrm{O}_{2}$, encontrou-se a imagem de subtração provocada pelo $\mathrm{N}_{2} \mathrm{O}$, o único elemento visível ao infra-vermelho, uma vez que tanto o $\mathrm{O}_{2}$ quanto o isoflurano já haviam se mostrado invisíveis (Grupos I e II). A presença de imagem de gás visível ao infra-vermelho obtida durante a descompressão abdominal no grupo III, demonstra claramente ser devido ao $\mathrm{N}_{2} \mathrm{O}$, pois se houvesse a interferência de vapor d'água ou de outros gases, este teria aparecido durante a descompressão abdominal dos grupos I e II.

Este estudo demonstra claramente a difusão do $\mathrm{N}_{2} \mathrm{O}$ para cavidades fechadas, em 45 minutos, em ratos submetidos a pneumoperitônio de $20 \mathrm{mmHg}$. Um autor ${ }^{7}$ concluiu em um modelo animal com porcos que o $\mathrm{N}_{2} \mathrm{O}$ utilizado na anestesia geral acumula-se no pneumoperitônio, e que em menos de 2 horas a cavidade intra-abdominal suína foi preenchida por $\mathrm{N}_{2} \mathrm{O}$ a $29 \%$, já suficiente para suportar combustão. O mesmo autor preconizou o escape contínuo dos gases, com reposição de $\mathrm{CO}_{2}$ fresco, em velocidade de 4 a 8 vezes por hora o volume inicial injetado de $\mathrm{CO}_{2}$ para insuflação da cavidade a $12 \mathrm{mmHg}^{20}$. O método empregado pelo referido autor foi coleta de gás da cavidade peritoneal do porco de 10 em $10 \mathrm{mi}-$ nutos e detecção por cromatografia gasosa. Este método implica coleta de gases em seringas de propileno, apresentando limitações e dificuldades de interpretação, pois os gases sofrem difusão para o meio ambiente, o que pode alterar o resultado final ${ }^{21}$.

O método da filmagem infra-vermelha foi um marco no estudo da exposição ocupacional aos agentes inalatórios e pode ser utilizado em pesquisa de cirurgia videolaparoscópica ou em qualquer outra pesquisa em Anestesiologia que envolva análise macroscópica de gases e sua semi-quantificação. Pode ser realizado em tempo contínuo, não necessitando coleta de amostras gasosas intra-peritoneais. Afilmagem é instantânea, ficando os dados gravados para posterior análise, não dependendo de laboratório e nem do fator tempo que pode vir a comprometer os resultados finais da análise.

Apesar de seu potencial para uso em laparoscopia como agente inalatório ou no próprio pneumoperitônio ${ }^{13}$, alguns pontos críticos e discutíveis persistem quanto ao seu uso em anestesia, principalmente em relação a contribuição para distensão de alças, apesar deste efeito ter sido contestado por diversos estudos mais recentes ${ }^{22}$. Em nosso estudo não houve aumento da pressão intra-abdominal, apesar da difusão do $\mathrm{N}_{2} \mathrm{O}$ para a cavidade intra-peritoneal. Outra preocu- 
pação é o potencial de formar misturas comburentes ${ }^{21,23,24}$, mas esta discussão se restringe a cirurgias nas quais ocorre lesão de alça intestinal com extravasamento de gases .

O uso do $\mathrm{N}_{2} \mathrm{O}$ vem sendo preconizado em cirurgias laparoscópicas por alguns autores, principalmente em colecistectomias

${ }^{13}$ e em cirurgias em que não ocorre extravasamento de gases intestinais para a cavidade. $\mathrm{O} \mathrm{N}_{2} \mathrm{O}$, usado como gás intracavitário para cirurgias laparoscópicas, apresentou menor incidência de alterações hemodinâmicas e ventilatórias ${ }^{25}$ quando comparado ao $\mathrm{CO}_{2}$, não tendo provocado disritmias ${ }^{26-29}$, prejuízo do campo cirúrgico e nem aumento da incidência de náuseas e vômitos no período pós-operatório, proporcionando ainda menor consumo de opióides ${ }^{30}$.

Pelos dados obtidos nesta pesquisa, conclui-se que o óxido nitroso por via inalatória a $66 \%$, administrado durante 45 minutos, difundiu-se para a cavidade peritoneal de ratos submetidos a pneumoperitônio com $20 \mathrm{mmHg}$ de ar ambiente, já que foi detectado macroscopicamente por filmagem no espectro infra-vermelho, sem que se tenha verificado aumento da pressão intra-abdominal nos 3 grupos estudados.

\section{Macroscopic Infrared Analysis of Inhaled Nitrous Oxide Diffusion to Abdominal Cavity in Rats submitted to Pneumoperitoneum}

Daniel Colman, M.D.; Marcos Leal Brioschi, M.D.; Mário Cimbalista Júnior, M.D.; Elizabeth Mila Tambara, TSA, M.D.; Maria Célia Barbosa Fabrício de Melo, TSA, M.D.; Leonardo Pimpão Blume, M.D.

\section{INTRODUCTION}

Nitrous oxide $\left(\mathrm{N}_{2} \mathrm{O}\right)$ is the oldest inhaled agent and is widely used worldwide ${ }^{1}$. Its properties, especially its low solubility ${ }^{2}$, give it special and desirable pharmacokinetic properties for an inhaled agent. Uptake, distribution and elimination processes are very fast. It has mild side-effects, and minor cardiovascular and respiratory repercussions should be emphasized $^{3,4}$. In addition, it is poorly metabolized.

Structurally, $\mathrm{N}_{2} \mathrm{O}$ is a tri-atomic asymmetric molecule with characteristics of major infrared irradiation absorption and emission ${ }^{5}$ with maximum absorption at $4.5 \mu \mathrm{m}$. For being used in high concentrations and widely used in pediatric anesthesia ${ }^{6}$, it has been chosen as a model for macroscopic studies of gases involved in anesthesiologists' occupational exposure. This way, it adds a new dimension to the study of anesthetic gases dispersion in the operating center and to the evaluation of OR's air drainage systems ${ }^{7}$. It also helps understanding the correct facial mask adaptation to prevent leakage and the development of educational material, acting also as an indicator of gases and vapors lost in the environment, both quantitatively and qualitatively ${ }^{8}$.
There are some controversies about the use of $\mathrm{N}_{2} \mathrm{O}$ in anesthesiology: thanks to its higher solubility as compared to nitrogen ${ }^{9}$, it is now playing the role previously plaid by nitrogen in body cavities filled with gases (such as middle ear) ${ }^{10-12}$, increasing both in volume and pressure when inside the cavity. Although its potential usefulness for laparoscopy as inhaled agent or in the pneumoperitoneum itself ${ }^{13}, \mathrm{~N}_{2} \mathrm{O}$ may contribute for intestinal loops distension ${ }^{14}$, although this effect has been questioned by more recent studies. Spivak et al. have not observed significant differences between the use of $\mathrm{N}_{2} \mathrm{O}$ or atmospheric air with regards to surgical field conditions in laparoscopic surgeries ${ }^{15}$.

This study aimed at macroscopically evaluating nitrous oxide diffusion to the peritoneal cavity by filming in the infrared spectrum rats submitted to pneumoperitoneum with 20 $\mathrm{mmHg}$ room air pressure.

\section{METHODS}

This experimental protocol has been approved by the Biological and Health Sciences Center, Pontifícia Universidade Católica, Paraná (CCBS-PUC/PR), was carried out according to Colégio Brasileiro de Experimentação Animal (COBEA) ethical principles and was filed with the Infrared Images Research Group of the National Research Council PUC/PR.

The experiment was performed with 15 male Wistar rats (Rattus norvegicus albinus, Rodentia mammalia), aged 120 to 153 days (mean 135.9 days), which received standardized feed and water ad libitum up to 12 hours before anesthesia.

The experiment was performed in the Medullary Injury and Experimental Trauma Lab, PUC/PR. Minor thermal variation was accepted, room temperature was maintained in $20^{\circ} \mathrm{C}$ and relative air humidity in $75 \%$, both checked by dry and wet bulb thermo-hygrometer (Incotherm, Br). Heat losses by convection were minimized by maintaining windows and doors closed and minimal movement around animals. Airflow was controlled with a digital rotation blade anemometer model HHF 300 A (Omega Engineering, Inc.) placed $10 \mathrm{~cm}$ away from animals and maintaining airflow velocity below 0.2 $\mathrm{m} . \mathrm{s}^{-1}$, which is the transition value between heat loss by free and forced convection ${ }^{16}$.

Anesthesia was induced and maintained with the studied agents: sodium thiopental, isoflurane and isoflurane associated to $66 \% \mathrm{~N}_{2} \mathrm{O}$, being inhaled anesthetic agents administered through facial cone with oxygen and universal vaporizer with $3 \mathrm{~L}$. $\mathrm{min}^{-1}$ gases fresh flow.

Animals were maintained in Guedel's anesthetic plane degree III. Monitoring consisted of checking for the presence of reflexes, respiratory rate, mucosal color ${ }^{17}$ and intra-abdominal pressure changes. Animals were divided in 3 groups:

- Group I - intravenous control: anesthetized with peritoneal thiopental and facial cone with 3 L. $\min ^{-1}$ oxygen; 
- Group II - inhaled control: anesthetized with $1.2 \%$ isoflurane and facial cone with 3 L. min $^{-1}$ oxygen;

- Group III - nitrous oxide: anesthetized with $0.6 \%$ isoflurane and facial cone with $1 \mathrm{~L} \cdot \mathrm{min}^{-1}$ oxygen associated to 2 L. $\min ^{-1} \mathrm{~N}_{2} \mathrm{O}(66.6 \%)$.

A "T" shaped plastic tube was coupled to universal gases vaporizer outlet to bypass the flow to two directions: one to the facial cone in contact with the animal for anesthetic induction and maintenance, and the other to control anesthetic gases and filming.

To obtain pneumoperitoneum ${ }^{18}$ with $20 \mathrm{mmHg}$ intra-abdominal pressure, peritoneal cavity was punctured with $22 \mathrm{G}$ teflon catheter connected to a previously gaged anerometer system (Welch Allyn, Tycos ${ }^{\circledR}$, Arden, USA). System remained coupled to abdominal cavity allowing continuous intra-abdominal pressure monitoring. Once the desired pressure was reached, inflation was interrupted. Forty-five minutes after pneumoperitoneum was deflated by a new abdominal puncture with $16 \mathrm{G}$ teflon catheter and gas leaving the abdominal cavity was filmed within the infrared spectrum between 3.5 to $5 \mu \mathrm{m}$. This second puncture aimed at obtaining a faster emptying. During abdominal decompression, anesthetic gases administration was withdrawn to prevent $\mathrm{N}_{2} \mathrm{O}$ contamination during filming.

Thermovision AGEMA 550 (FLIR System ${ }^{\text {TM }}$, Sweden) radiometer was used to capture spectral electromagnetic waves range between 3.5 and $5 \mu \mathrm{m}$, that is, short infrared spectrum waves. Maximum spatial resolution obtained was 0.1 to $0.2 \mathrm{~mm}$.

Naturally emitted infrared irradiation by objects in the environment is captured and converted into electric signal by a PtSi detector cooled by liquid nitrogen (steerling cycle), This signal is processed in a numeric spreadsheet with 76 thousand absolute temperature points and gaged by frame, instantaneously represented by thermal image with resolution of $320 \times 240$ pixels and thermal sensitivity above $0.1{ }^{\circ} \mathrm{C}$. Tri-molecular gases, including $\mathrm{N}_{2} \mathrm{O}$, have the physical property of energy absorption and emission in the infrared range 4,19 , which makes them visible at infrared when placed between a heat-emitting source and radiometer.

An acrylic box with $40^{\circ} \mathrm{C}$ water was used in our study as the heat emitting source for nitrous oxide visualization at infrared.

Radiometer was assembled in a vertical support $1 \mathrm{~m}$ away from the acrylic box and directly focused on animals' ventral surface forming a $60^{\circ}$ angle with the teflon catheter during filming of abdominal decompression. For control purposes, gases being administered via facial cone were also filmed through the " $T$ " outlet of the anesthesia system, as shown in figure 1.

Images were processed by a $750 \mathrm{MHz}$ Pentium III computer coupled to a PCMCIA board. Using a specific program, ThermaCAM $^{\mathrm{TM}}$ Researcher 2001, FLIR Systems (Sweden), captured images were recorded at 7 frames per second throughout abdominal decompression, in the same above-described environmental conditions. All images were

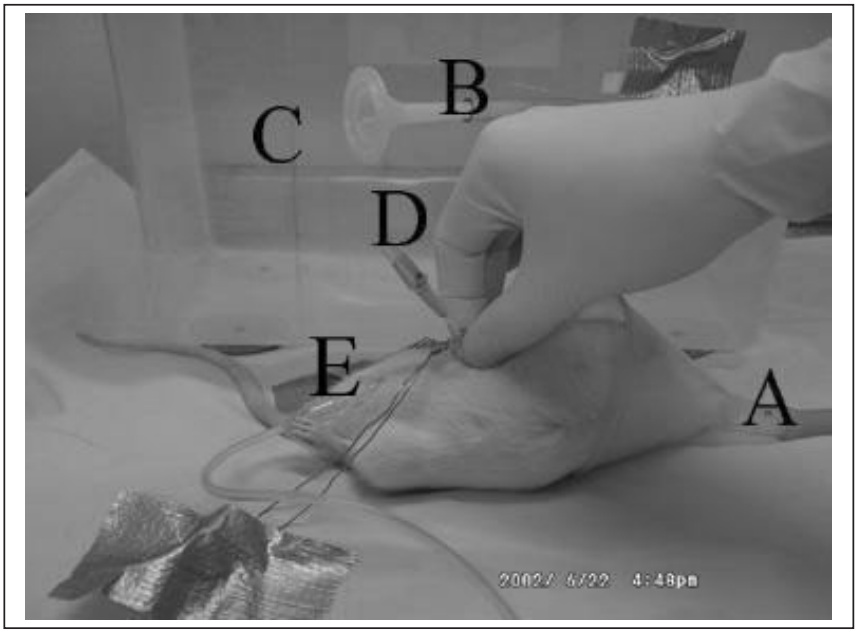

Figure 1 - Experiment Assembly

A) Facial cone through which anesthetic agent was administered to animals; B) Control of anesthetic gases administered to animals; C) Acrylic box filled with warm water and acting as a support for infrared filming; D) Teflon catheter for abdominal decompression; E) Anerometer system connected to abdominal cavity for pneumoperitoneum and intra-abdominal pressure monitoring

represented by infrared thermographs in a video monitor and recorded in CD-Rom for further analysis by the program.

Images were plotted using thermal range between $50^{\circ} \mathrm{C}$ and $20{ }^{\circ} \mathrm{C}$, level temperature of $42{ }^{\circ} \mathrm{C}$ and continuous "RAINBOW900" color palette, in which white, red, yellow, green, blue and black represented a decreasing temperature gradient, equally distributed throughout the scale from hottest to coldest and maintained until experiment completion. For black and white printing purposes, images were treated with Adobe Photoshop 5.0 software through which colors were filtered in the red palette followed by the application of grey shades for a better contrast among researched elements.

For semi-quantitative analysis of infrared-visible gases, temperature differential between what animals were inhaling (item B, Figure 1) and what left abdominal cavity was calculated (item D, Figure 1).

\section{RESULTS}

Nitrous oxide $(66 \%)$ is visible to infrared through the method described and recorded in thermographs, where yellowish colors represent heat emission by the acrylic support filled with warm water, and greenish colors (colder) represent the energy absorbed by $\mathrm{N}_{2} \mathrm{O}$ forming a visible contrast at infrared filming, as shown in figure 2.

There were no infrared-visible gas traces in thermographs obtained for Group I during macroscopic filming of gases from the facial cone $\left(100 \% \mathrm{O}_{2}\right)$, as shown in figure $3 . \mathrm{O}_{2}$ was invisible at infrared. There were also no gas traces images in thermographs obtained during macroscopic filming of intra-abdominal cavity gases during decompression, as shown in figure 4. 


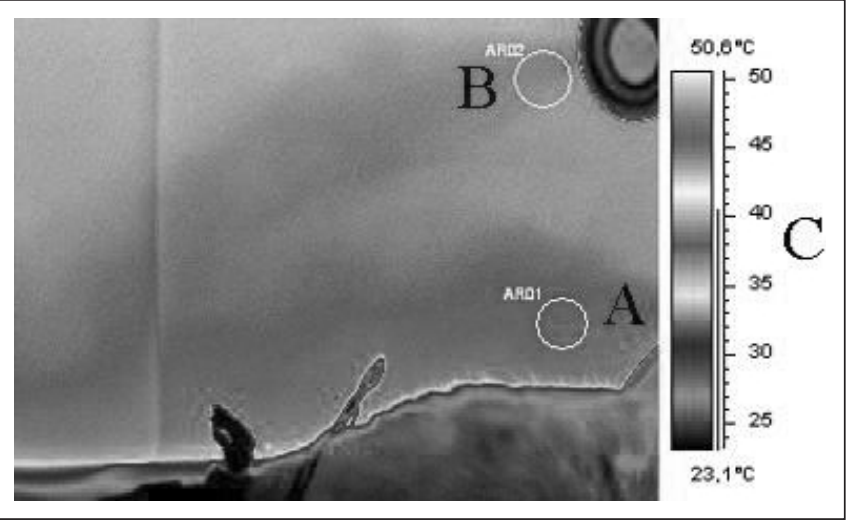

Figure 2 - A) Facial Cone Gases; B) Gases from the control of A; C) Color palette. In A and B gases are visible at infrared

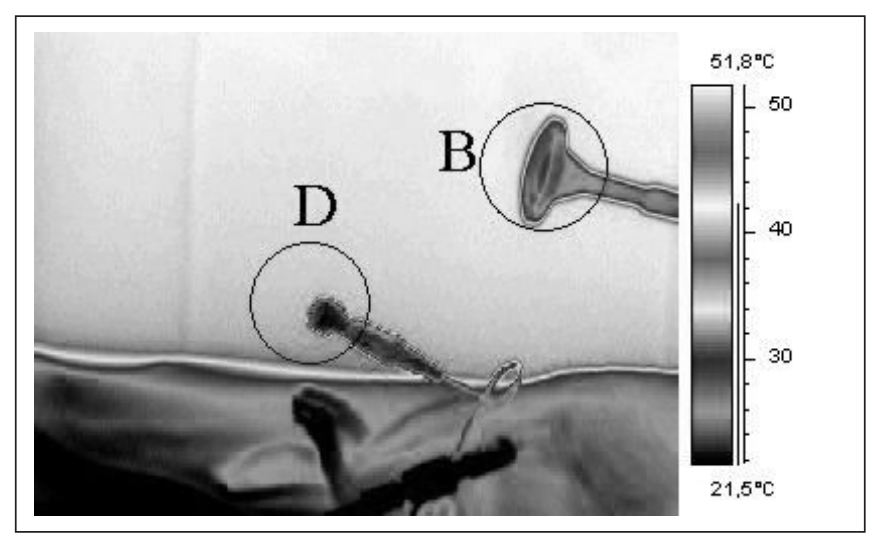

Figure 3 - Pre Abdominal Decompression

B) Gas not visible at infrared

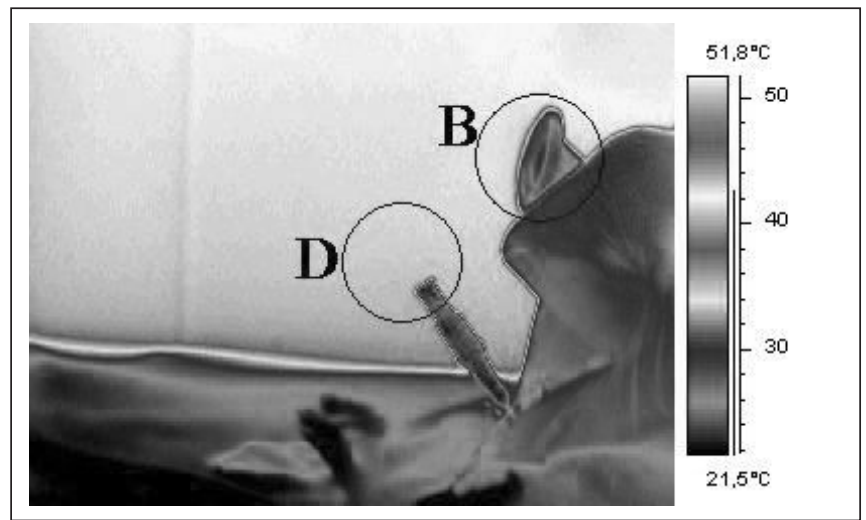

Figure 4 - During Abdominal Decompression $B$ and $D$ are invisible to infrared
There were no infrared-visible gas traces in thermographs obtained for Group II during macroscopic filming of gases from the facial cone $\left(100 \% \mathrm{O}_{2}\right.$ carrying $1.2 \%$ isoflurane). In our study, with the method and spectral range used, isoflurane was invisible to infrared. There were also no gas traces images in thermographs obtained during macroscopic filming of intra-abdominal cavity gases during decompression. Thermograph patterns in Group II follow figures 3 and 4 patterns.

There have been infrared-visible gas traces in thermographs obtained for Group III during macroscopic filming of gases from the facial cone $\left(33.4 \% \mathrm{O}_{2}\right.$ associated to $66.6 \% \mathrm{~N}_{2} \mathrm{O}$ and $0.6 \%$ isoflurane). This clearly shows that $\mathrm{N}_{2} \mathrm{O}$ is visible in thermographs obtained in the infrared range as shown in figure 2. There have been also gases trace images in thermographs obtained during macroscopic filming of intra-abdominal cavity gases during decompression, as shown in figures 5 and 6 . Temperature differential between $\mathrm{B}$ in figure 2 and $\mathrm{D}$ in figures 5 and 6 was close to zero, suggesting that $\mathrm{N}_{2} \mathrm{O}$ concentration leaving the cavity was close to the inspired fraction, that is, $66 \%$.

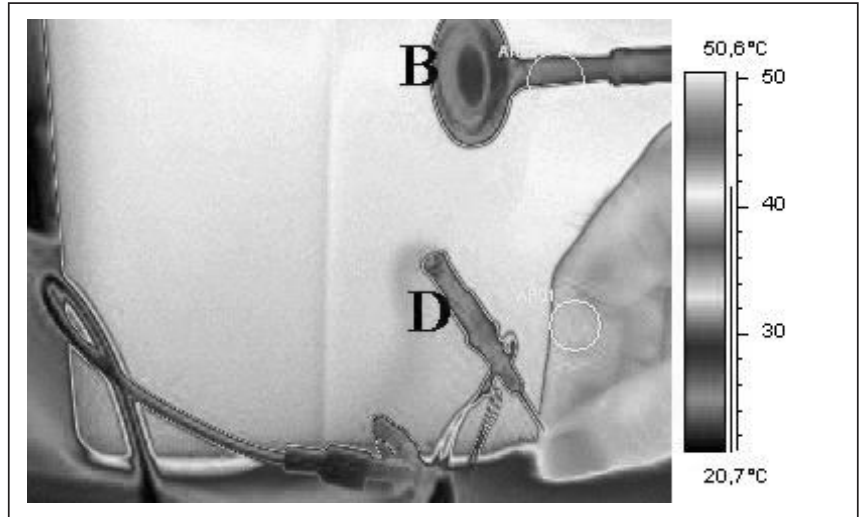

Figure 5 - During Group III Abdominal Decompression

B) Pattern of gas not visible at infrared because $\mathrm{N}_{2} \mathrm{O}$ administration was withdrawn during abdominal decompression; D) Pattern of gas visible to infrared

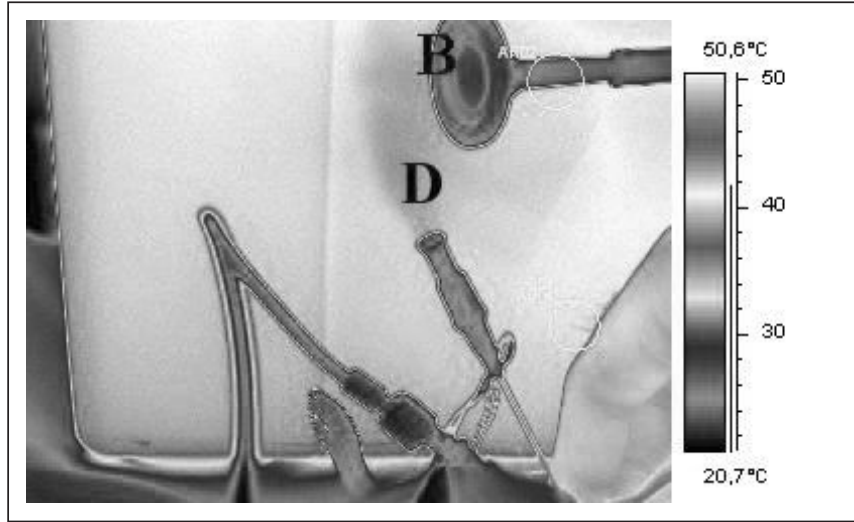

Figure 6 - Abdominal Decompression with Pattern of Gas Visible at Infrared, Coming from Peritoneal Cavity (D)

Revista Brasileira de Anestesiologia

Vol. 54, N 2, Março - Abril, 2004 
This shows the diffusion of nitrous oxide used in inhaled anesthesia to the abdominal cavity of rats submitted to pneumoperitoneum with $20 \mathrm{mmHg}$ room air.

Intra-abdominal pressure was maintained stable in $20 \mathrm{mmHg}$ throughout the study for all Groups.

\section{DISCUSSION}

Most gases and vapors with bipolar molecular structure absorb infrared energy. If the gas is placed between an infrared-emitting object and a radiometer, gas will absorb infrared irradiation and this absorbed energy will be translated into temperature decrease, being visible at thermograph analysis ${ }^{19}$. This method allows for the visualization of gases invisible to human eye and has been used for the study of most popular inhaled agents pollution levels. It allows for the study of pollutant gases and vapors dispersion during our professional activity. A study has evaluated pollution levels in pediatric service rooms and has shown the importance of accurately handling facial masks to prevent pollutant anesthetic gases losses to the environment. It has concluded that this method, in addition to allowing macroscopic gas diffusion analysis, also provides a semi-quantitative analysis correlated to data obtained from the gases analyzer ${ }^{19}$. The same author has concluded that pollutant gases are poorly eliminated from the OR during ventilation. In our study, in addition to macroscopically analyzing inhaled $\mathrm{N}_{2} \mathrm{O}$ diffusion to pneumoperitoneum, we could conclude that its concentration was close to the inspired fraction through images subtraction analysis.

Atmosphere has some energy emission and absorption properties. Gas and vapor molecules vibrate in certain frequencies ${ }^{5}$ which, when reached, absorb an energy photon, allowing the gas to be contrasted when passing between emitting source and radiometer.

Monoatomic gases, such as neon, have only electronic transitions, while biatomic gases, such as $\mathrm{O}_{2}$ and $\mathrm{N}_{2}$, have no electric dipole moment, thus not absorbing or emitting significant energy in environmental conditions and being considered invisible at infrared ${ }^{5}$. This has been observed in our study because there has been no image whatsoever of infrared-visible gas when $\mathrm{O}_{2}$ was used in Group I.

Pneumoperitoneum was achieved with room air which is knowingly invisible to infrared.

Asymetric diatomic molecules, such as $\mathrm{NO}$ and $\mathrm{CO}$ emit and absorb infrared energy, but in a very weak manner in environmental conditions. Triatomic molecules, however, such as $\mathrm{N}_{2} \mathrm{O}, \mathrm{SO}_{2}, \mathrm{H}_{2} \mathrm{O}$ and $\mathrm{CO}_{2}$, have strong energy emission and absorption trends. These gases may impair the interpretation of image components because the radiometer used in our study has an observation window in the range of 3.5 to $5 \mu \mathrm{m}$, while such molecules have windows with absorption peaks in the following ranges ${ }^{5}: \mathrm{CO}_{2}(2,0 ; 2,7,4,3$; $15 \mu \mathrm{M}) ; \mathrm{H}_{2} \mathrm{O}(1,4 ; 1,9 ; 2,7 ; 6,320 \mu \mathrm{M})$ and $\mathrm{N}_{2} \mathrm{O}(4,5 \mu \mathrm{M})$. This phenomenon could be controlled in our study by using room air for pneumoperitoneum, which is knowingly invisible to infrared.
Knowing that $\mathrm{O}_{2}$ is invisible to infrared, images obtained with isoflurane in Group II (as control of what was being vaporized), have shown that this agent was also invisible in the conditions of our study. Since there has been no infrared-visible gas traces at abdominal decompression of Group II animals, it is unlikely that water vapor from the peritoneal serosa in the abdominal cavity would influence results found by this study. As to Group III receiving inhaled $\mathrm{N}_{2} \mathrm{O}$ with isoflurane and $\mathrm{O}_{2}$, the only visible element at infrared was the subtraction image caused by $\mathrm{N}_{2} \mathrm{O}$, since both $\mathrm{O}_{2}$ and isoflurane had already shown to be invisible (Groups I and II). The presence of infrared-visible image at abdominal decompression in Group III has clearly shown that this was due to $\mathrm{N}_{2} \mathrm{O}$, because if there was water vapor or other gases influence, this would had been shown during abdominal decompression of Groups I and II.

This study has clearly shown $\mathrm{N}_{2} \mathrm{O}$ diffusion to closed cavities in 45 minutes, in rats submitted to $20 \mathrm{mmHg}$ pneumoperitoneum. An author ${ }^{7}$ has concluded in an animal model with swine, that $\mathrm{N}_{2} \mathrm{O}$ used in general anesthesia would build up in the pneumoperitoneum, and that in less than 2 hours, swine intra-abdominal cavity had been filled with $29 \% \mathrm{~N}_{2} \mathrm{O}$, enough to undergo combustion. The same author has preconized continuous gas escape with replacement with fresh $\mathrm{CO}_{2}$ in a rate of 4 to 8 times/hour the initial $\mathrm{CO}_{2}$ injected volume to inflate cavity at $12 \mathrm{mmHg}^{29}$. The author has collected gas from swine peritoneal cavity at 10-minute intervals detected by gas chromatography. This method implies gases collection in propylene syringes and has limitations and interpretation difficulties, since gases are diffused to the environment and may affect final results ${ }^{21}$.

Infrared filming method was a landmark in the study of occupational exposure to inhaled agents and may be used in laparoscopic surgery research or in any other Anesthesiology research involving macroscopic gases analysis and semi-quantification. It may be continuously performed with no need for intra-peritoneal gases sample collection. Filming is instantaneous and data are recorded for further analysis, not depending on laboratories or on time, which could interfere with final results.

Although its potential use in laparoscopy as inhaled agent, or even in the pneumoperitoneum itself ${ }^{13}$, some critical and questionable issues still remain as to its use in anesthesia, especially its contribution in distending intestinal loops, although this defect has been contested by more recent studies ${ }^{22}$. There has been no intra-abdominal pressure increase in our study, in spite of $\mathrm{N}_{2} \mathrm{O}$ diffusion to the intra-peritoneal cavity. Another concern would be the potential formation of carburetant blends ${ }^{21,23,24}$, but this discussion is restricted to surgeries where there is intestinal loop injury with gases leakage.

$\mathrm{N}_{2} \mathrm{O}$ has been preconized for laparoscopic surgeries by some authors, especially for cholecystectomies ${ }^{13}$ and surgeries where there is no intestinal gases leakage to the cavity. $\mathrm{N}_{2} \mathrm{O}$ as intracavitary gas for laparoscopic surgeries has shown a lower incidence of hemodynamic and ventilatory changes ${ }^{25}$ as compared to $\mathrm{CO}_{2}$ and has not promoted arhythmias ${ }^{26-29}$, surgical field difficulties or an increase in the incidence of 
postoperative nausea and vomiting, in addition to allowing lower consumption of opioids ${ }^{30}$.

From the data obtained in our study, one may conclude that $66 \%$ inhaled nitrous oxide administered for 45 minutes has diffused to the abdominal cavity of rats submitted to pneumoperitoneum with $20 \mathrm{mmHg}$ room air, since it has been macroscopically detected by infrared filming without intra-abdominal pressure increase in all studied groups.

\section{REFERÊNCIAS - REFERENCES}

01. Nocite JR - Óxido nitroso: perspectivas para o ano 2000. Rev Bras Anestesiol, 1993;43:157-158.

02. Eger II El, Larson Jr CP - Anaesthetic solubility in blood and tissues: values and significance. Br J Anaesth, 1964;36:140-144.

03. Saidman LJ, Eger II EI - Effect of nitrous oxide and of narcotic premedication on the alveolar concentration of halothane required for anesthesia. Anesthesiology, 1964;25:302-306.

04. Thornton JA, Fleming JS, Goldberg AD et al - Cardiovascular effects of $50 \%$ nitrous oxide and $50 \%$ oxygen mixture. Anaesthesia, 1973;28:484-489.

05. Moore PM, Maldague XPV - Infrared and Thermal Testing. American Society for Nondestructive Testing, $3^{\text {rd }}$ Ed, John Wiley \& Sons, 1988;3:180-184;580-586.

06 . Whitcher C, Piziali R - Monitoring occupational exposure to inhaIation anesthetics. Anesth Analg, 1977;56:778-785.

07. Carlsson P, Ljungqvist B, Neikter K - Thermocamera studies of gases and vapours. Br J Ind Med, 1982;39:300-305.

08. Allander C, Carlsson P, Hallen B et al - Thermocamera, a macroscopic method for the study of pollution with nitrous oxide in operating theaters. Acta Anaesthesiol Scand, 1981;25:21-24.

09. Saidman LJ, Eger El - Change in cerebrospinal fluid pressure during pneumoencephalography under nitrous oxide anesthesia. Anesthesiology, 1965;26:67-72

10. Patterson ME, Bartlett PC - Hearing impairment caused by intratympanic pressure changes during general anesthesia. Laryngoscope, 1976;86:399-404

11. Man A, Segal S, Ezra S - Ear injury caused by elevated intratympanic pressure during general anesthesia. Acta Anaesthesiol Scand, 1980;24:224-226.

12. Katayama M, Panhoca R, Vieira JL et al - Alterações no ouvido médio induzidas pelo óxido nitroso e suas implicações clínicas. Rev Bras Anestesiol, 1992;42:397-404

13. Katayama M, Vieira JL, Campos JL et al - Óxido nitroso: uma boa opção como gás para pneumoperitôneo nas colecistectomias por videolaparoscopia sob anestesia geral. Rev Bras Anestesiol, 1996;46:78-87

14. Scheinin R, Lindgren L, Scheinin TM - Peroperative nitrous oxide delays bowel function after colonic surgery. $\mathrm{Br} \mathrm{J}$ Anaesth, 1990;64:154-158.

15. Spivak H, Nudelman I, Fuco $\mathrm{V}$ et al - Laparoscopic extraperitoneal inguinal hernia repair with spinal anesthesia and nitrous oxide insuflation. Surg Endosc, 1999;13: 1026-1029.

16. Gagge AP, Nishi $Y$ - Heat Exchange between Human Skin Surface and Thermal Environment, em: Lee D - Handbook of Physiology. Reactions to Environmental Agents. American Physiological Society, 1977;69-92.

17. Dripps RD - Evaluation of the Response to Anesthetics: the Signs and Stages, em: Introduction to Anaesthesia. The Principle of Safe Practice. $5^{\text {th }}$ Ed, WB Saunders, 1977;233.
18. Cunningham AJ - Anesthetic implications of laparoscopic surgery. Yale J Biol Med, 1998;71:551-578

19. Eleftheriadis E, Kotzampassi K, Papanotas K et al - Gut ischemia, oxidative stress and bacterial translocation in elevated abdominal pressure in rats. World J Surg, 1996;20:11-16.

20. Diemunsch PA, Torp KD, Van Dorsselaer T et al - Nitrous oxide fraction in the carbon dioxide pneumoperitoneum during laparoscopy under general inhaled anesthesia in pigs. Anesth Analg 2000;90:951-953.

21. Diemunsch PA, Van Dorsselaer T, Torp KD et al - Calibrated pneumoperitoneal venting to prevent $\mathrm{N}_{2} \mathrm{O}$ accumulation in the $\mathrm{CO}_{2}$ pneumoperitoneum during laparoscopy with inhaled anesthesia: an experimental study in pigs. Anesth Analg, 2002;94:1014-1018.

22. Hunter JG, Staheli J, Oddsdottir M et al - Nitrous oxide pneumoperitoneum revisited. Is there a risk of combustion? Surg Endosc, 1995;9:501-504.

23. Taylor E, Feinstein R, White PF et al - Anesthesia for laparoscopic cholecystectomy. Is nitrous oxide contraindicated? Anesthesiology, 1992;76:541-543

24. Neuman GG, Sidebotham G, Negoianu E et al - Laparoscopy explosion hazards with nitrous oxide. Anesthesiology, 1993;78: 875-879.

25. Corall IM, Elias JA, Strunin L - Laparoscopy explosion hazards with nitrous oxide. Br Med J, 1975;4:5991:288.

26. Katayama M, Campos JL, Cardoso PRO et al - Anestesia geral para colecistectomia laparoscópica: efeito do óxido nitroso sobre a ventilação pulmonar. Rev Bras Anestesiol, 1993;43: 313-321.

27. Johannsen G, Andersen M, Juhl B - The effect of general anaesthesia on the hemodynamic events during laparoscopy with $\mathrm{CO}_{2}$ insuflation. Acta Anaesthesiol Scand, 1989;33:132-136.

28. Marschall RL, Jebson PJR, Davie IT et al - Circulatory effects of peritoneal insufflation with nitrous oxide. $\mathrm{Br} \mathrm{J}$ Anaesth, 1982;44:1183-1187.

29. Ooka T, Kawano $Y$, Kosaka $Y$ et al - Blood gas changes during laparoscopic cholecystectomy: comparative study of $\mathrm{N}_{2} \mathrm{O}$ pneumoperitoneum and $\mathrm{CO}_{2}$ pneumoperitoneum. Masui, 1993;42:398-401.

30. Minoli G, Terruzzi V, Spinzi GC et al - The influence of carbon dioxide and nitrous oxide on pain during laparoscopy: a double-blind controlled trial. Gastrointestinal Endoscopy, 1982;28: 173-175.

\section{RESUMEN}

Colman D, Brioschi ML, Cimbalista Jr M, Tambara EN, Melo MCBF, Blume LP - Análisis Macroscópica Infrarroja de la Difusión del Óxido Nitroso Vía Inhalatoria para la Cavidad Abdominal, en Ratones Sometidos a Pneumoperitoneo

JUSTIFICATIVA Y OBJETIVOS: El óxido nitroso $\left(\mathrm{N}_{2} \mathrm{O}\right)$, por ser una estructura tri-atómica asimétrica, toma características de alta emisión y absorción de energía en el espectro infrarrojo, con un pico característico de absorción en 4,5 $\mu \mathrm{m}$, lo que lo hace visible al infrarrojo corto, cuando contrastado con una fuente emisora de calor (resguardo caliente). Diversos autores han descrito la difusión del $\mathrm{N}_{2} \mathrm{O}$ para cavidades cerradas por métodos como cromatografia gaseosa y analizador de gases, que no permiten un estudio macroscópico detallado del gas. El presente estudio tubo como objetivo la filmación macroscópica en el espectro infrarrojo de la difusión de $\mathrm{N}_{2} \mathrm{O}$, utilizado en anestesia inhalatoria, para la cavidad peritoneal de ratones sometidos a pneumoperitoneo de $20 \mathrm{mmHg}$ con aire ambiente. 
MÉTODO: Los animales fueron divididos en tres grupos, de acuerdo con el anestésico utilizado: I-Control venoso: tiopental intra-peritoneal; II- Control inhalatorio: isoflurano a 1,2\% en $\mathrm{O}_{2}$ 100\%; III- Óxido Nitroso: $\mathrm{N}_{2} \mathrm{O} 66 \%$ en oxígeno e isoflurano a $0,6 \%$. Los termogramas provenientes de la descompresión abdominal fueron obtenidos, por medio de un radiómetro AGEMA 550 filmados a 7 cuadros por segundo.

RESULTADOS: EI $\mathrm{N}_{2} \mathrm{O}$ se demostró visible al infrarrojo. En el momento de la descompresión abdominal, no hubo en los grupos I y II termogramas con rastros de gases visibles al infrarrojo. Hubo, todavía, rastros de gases visibles al infrarrojo en el grupo III.

CONCLUSIONES: Se concluye que el óxido nitroso inhalatorio a $66 \%$ se difundió para la cavidad peritoneal de ratones sometidos a pneumoperitoneo de $20 \mathrm{mmHg}$ con aire ambiente, sin aumento de la presión intra-abdominal. 